\title{
The equilibrium gap method : a two-scales approach
}

\author{
F. Amiot ${ }^{1, a}$
}

FEMTO-ST Institute, CNRS-UMR 6174/UFC/ENSMM/UTBM, 24 chemin de l'Épitaphe, F-25030 Besançon, France

\begin{abstract}
Optical full-field measurement methods are increasingly used in the field of experimental mechanics, and several techniques have been developped to make the most of the redundancy of the measured fields. However, the number of modelling parameters that can be retrieved is strongly connected to the noise level corrupting the measured fields. Focusing on the techniques based on the construction of a statically admissible field from the experimental data, a two-scales approach is proposed. It relies on the fact that, using a finite-element description of the struture, the larger the number of degrees of freedom to be measured, the more degraded by noise. It is therefore proposed to use displacement fields measured at two different scales. This paper describes the way the macro displacement fields basis is built.
\end{abstract}

\section{Introduction}

Optical full-field measurement methods are increasingly used in the field of experimental mechanics. These techniques are now well established so that extensive knowledge about the mechanisms driving the measurements quality is now available (for DIC measurements, see [1]). These methods provide a very large amount of data, so that different techniques have been proposed to identify material properties using redundant or full-field kinematic data [2]. However, the number of parameters that can be identified using these methods is directly related to the noise level degrading the measured field. This can lead to either significant errors on the description or a poor resolution of the structure under scrutiny, especially when this is extremely heterogeneous and/or subjected to an unknown loading field (i.e. for MEMS devices [3]). The equilibrium gap method is first recalled, before a two-scales approach is presented. The projection conditions to be satisfied by the macro displacement field basis are then derived, and a procedure to build the projection operator is proposed. The method is then illustrated on an example.

\section{Mono-scale equilibrium gap method}

One considers an elastic body, whose displacement is measured at several points $\mathbf{x}$. For the sake of brevity, only cantilever beams under transverse loading are considered in the sequel. One supposes a stiffness field $C(\mathbf{x})$ described by a finite number of functions $d_{i}(\mathbf{x})$ and a scaling parameter $C_{0}$ :

$$
C(\mathbf{x})=C_{0} \sum_{i=1}^{N} \mathbf{D}_{i} d_{i}(\mathbf{x})
$$

where $N$ is the number of used functions and $\left\{\mathbf{D}_{i}\right\}_{i=1 \ldots N}$ the projection of the stiffness field onto the defined basis. The structure is subjected to a loading field $Q_{m}(\mathbf{x})$, which is also described by a finite

a e-mail: fabi en. ann ot @ento-st. fr 
number $S$ of functions $\psi_{m s}(\mathbf{x})$

$$
Q_{m}(\mathbf{x})=\sum_{s=1}^{S} \mathbf{F}_{m s} \psi_{m s}(\mathbf{x})
$$

$\left\{\mathbf{L}_{m s}\right\}_{s=1 \ldots S}$ is the projection of the loading field onto the defined basis. This results in a out-of-plane displacement field $v_{m}(\mathbf{x})$ :

$$
v_{m}(\mathbf{x})=\sum_{k=1}^{K} U_{m k} \phi_{m k}(\mathbf{x})
$$

In the following, it will be assumed that the chosen basis are well suited to describe both stiffness, loading and displacement fields. The previously proposed equilibrium gap method ([5]) then relies on the finite element formulation of the direct problem : when the stiffness and loading fields are known (through their projections $\mathbf{D}$ and $\mathbf{L}_{m}$ ), the nodal displacement components $U_{m k}$ are computed by solving

$$
\mathcal{K}_{m} \mathbf{U}_{m}=\mathbf{L}_{m}
$$

The components of the stiffness operator $\mathcal{K}_{m}$ read

$$
\mathcal{K}_{m_{k, l}}=C_{0} \sum_{i=1}^{N} \mathbf{D}_{i} \int d_{i}(\mathbf{x}) \frac{\mathrm{d}^{2} \phi_{m k}(\mathbf{x})}{\mathrm{d} \mathbf{x}^{2}} \frac{\mathrm{d}^{2} \phi_{m l}(\mathbf{x})}{\mathrm{d} \mathbf{x}^{2}} \mathrm{~d} \mathbf{x}
$$

and

$$
\mathbf{L}_{m_{k}}=\sum_{s=1}^{S} \mathbf{F}_{m s} \int \psi_{m s}(\mathbf{x}) \phi_{m k}(\mathbf{x}) \mathrm{d} \mathbf{x}
$$

In order to identify the fields $C(\mathbf{x})$ and $Q_{m}(\mathbf{x})$ from measured displacement fields, and contrary to the resolution of the direct problem, the nodal displacement field $\mathbf{U}_{m}$ is here considered as "almost" known (i.e., measured)

$$
\mathbf{U}_{m} \simeq \widehat{\mathbf{U}_{m}}
$$

The equilibrium conditions (4) are then recast as

$$
\mathcal{M}_{m}\left(\widehat{\mathbf{U}_{m}}\right) \mathbf{S}=\mathcal{K}_{m}(\mathbf{S}) \widehat{\mathbf{U}_{m}}-\mathbf{L}_{m}(\mathbf{S})=\mathbf{R}
$$

where $\mathbf{S}$ is the concatenation of the stiffness parameters $\mathbf{D}$ and the loading parameters $\mathbf{F}_{m}$. The Eq.(8) is solved for the non-trivial $\mathbf{S}$ by minimization of the residual $\mathbf{R}$ when the number of nodal displacements used to write the equilibrium conditions is large compared to the number of parameters to identify. When the number of unknowns in $\mathbf{S}$ increases, the Eq.(8) is solved by singular value decomposition taking into account that under the assumption of linear behavior, the operator $\mathcal{M}_{m}$ is singular, and the solution $\mathbf{S}_{s o l}$ is obtained as the right singular vector associated to the least singular value [3]. The sensitivity of this last procedure to the measurement noise has been assessed and has been found to dramatically increase with the number of parameters to be identified, therefore limiting, for a given measurement quality, the achievable description level. This is a critical issue when the structures and/or the loadings are not well defined, for example when dealing with MEMS devices under environmental loadings.

Equation (8) then defines a statically admissible displacement field. Any norm of the residual $\mathbf{R}$ then provides a distance between the measured displacement field and its statically admissible projection. From its definition, finding a statically admissible displacement field is similar to finding the stiffness and the loading related by this statically admissible displacement field.

\section{Two-scales approach}

\subsection{Projection conditions}

As this is likely to result in a large number of parameters to be identified, the problem is to find a robust identification scheme. As one assumes that only displacement fields are measured, one will consider 
using different projections of the measured displacement field onto different basis, thus defining a "macro"-measured displacement field $v_{M}(\mathbf{x})$ :

$$
v_{M}(\mathbf{x})=\sum_{p=1}^{P} U_{M p} \phi_{M p}(\mathbf{x})
$$

with $P<K$. For a given structure, described by the sets $\left\{D_{n}, L_{m s}\right\}$, it is then worth noting that one has two ways of computing the displacement projection $\left\{U_{M p}\right\}$ :

- The first one mimics the projection of the measured displacement field onto the reduced kernel of displacement functions. The displacement field $v_{m}(\mathbf{x})$ is computed using the equilibrium conditions at the micro-scale (Eq. (4)) and projecting the obtained displacement field onto the "macro" displacement basis.

- The second way involves an homogenization procedure. Using a suitable macro stiffness matrix $\mathcal{K}_{M}$, the macro displacement fields satisfies some macro equilibrium conditions :

$$
\mathcal{K}_{M} \mathbf{U}_{M}=\mathbf{L}_{M}
$$

If the first way would be natural from the computing point of view, it is useless from the experimental one since it requires unachievable data. The problem is then to define some conditions on the basis to be used so that the second way will provide the same projected displacement field than the first way.

Let us define the projection operator $\mathcal{W}$ so that the function basis at the macro and micro scales are related through

$$
\phi_{M p}(\mathbf{x})=\sum_{k=1}^{K} \mathcal{W}_{p k} \phi_{m k}(\mathbf{x})
$$

$W$ is therefore defined by $P \times K$ coefficients. Starting with the first way, the displacement field at the macro scale $\mathbf{U}_{M}^{k \star}$ derived from a test displacement field at the micro scale $\mathbf{U}_{m}^{\star}$ is simply obtained by projection onto the macro basis. Minimizing a least-square norms yields

$$
\mathcal{W} \mathcal{H} \mathcal{W}^{t} \mathbf{U}_{M}^{k \star}=\mathcal{A}_{M} \mathbf{U}_{M}^{k \star}=\mathcal{W} \mathcal{H} \mathbf{U}_{m}^{\star}
$$

where $\mathcal{H}$ is defined by

$$
\mathcal{H}_{i j}=\int \phi_{m i}(\mathbf{x}) \phi_{m j}(\mathbf{x}) d x
$$

On the other hand, the stiffness matrix at the macro scale $\mathcal{K}_{M}$ is obtained by expressing the strain energy in the micro stiffness field and the macro displacement field. The definition (11) then yields

$$
\mathcal{K}_{M}=\mathcal{W} \mathcal{K}_{m} \mathcal{W}^{t}
$$

The macro loading term $\mathbf{L}_{M}$ is also obtained by writing the work of the micro loading field in the macro displacement field

$$
\mathbf{L}_{M}=\mathcal{W} \mathbf{L}_{m}=\mathcal{W} \mathcal{K}_{m} \mathbf{U}_{m}^{\star}
$$

The displacement field at the macro scale $\mathbf{U}_{M}^{S \star}$ is therefore defined as satisfying

$$
\mathcal{K}_{M} \mathbf{U}_{M}^{S \star}=\mathbf{L}_{M}
$$

The displacement field $\mathbf{U}_{M}^{k \star}$ is the one that is measured when using a reduced functions kernel (that is with a reduced noise sensitivity), whereas $\mathbf{U}_{M}^{s \star}$ satisfies the equilibrium conditions at the macro scale as long as these are satisfied at the micro scale. Finding an operator $\mathcal{W}$ so that

$$
\mathbf{U}_{M}^{k \star}=\mathbf{U}_{M}^{S \star}
$$


allows one to get equilibrium conditions at the macro scale (on the parameters at the micro scale) from displacement fields measured at the macro scale, so that these are less corrupted by the measurement noise. The condition (17) then yields

$$
\mathcal{W} \mathcal{K}_{m}\left(\mathcal{W}^{t} \mathcal{A}_{M}^{-1} \mathcal{W} \mathcal{H}-\mathcal{I}\right) \mathbf{U}_{m}=\mathcal{W} \mathcal{K}_{m} \cdot \mathbf{G}_{m}=\mathbf{0}
$$

The condition (18) is satisfied for any stiffness field if $\mathbf{G}_{m}$ belongs to stiffness operator nullspace $K_{m}^{\perp}$. As the stiffness operator is obtained without use of any a priori boundary condition on the displacement (since the latter are measured), $K_{m}^{\perp}$ is composed of the structure rigid-body motions. It is therefore straightforward to define the operator $\mathcal{R}_{\perp}$ so that

$$
\mathcal{R}_{\perp}^{t} \cdot \mathbf{G}_{m}=\mathbf{0}
$$

One therefore has to define the projection operator $\mathcal{W}$ satisfying Eq.(19) for some user-defined test displacement fields $\mathbf{U}_{m}^{\star}$.

\subsection{Projection operator computation}

Considering the singular value decomposition of $\mathcal{H}$

$$
\mathcal{H}=\mathcal{V} E \mathcal{V}^{t}
$$

the projection operator $\mathcal{W}$ is sought as

$$
\mathcal{W}=\mathcal{P} \mathcal{V}^{t}
$$

The problem turns then to find the operator

$$
\mathcal{G}=\mathcal{P}^{t}\left(\mathcal{P} \mathcal{E} \mathcal{P}^{t}\right)^{-1} \mathcal{P}
$$

satisfying

$$
\mathcal{V} \mathcal{E} V^{t} \mathbf{U}_{m}^{\star}=\mathbf{U}_{m}^{\star}+\mathcal{R} \mathbf{r}_{0}
$$

where $\mathcal{R} \mathbf{r}_{0}$ is a linear combination of the rigid body motions subspace. Defining $\mathcal{G}$ through its singular value decomposition

$$
\mathcal{G}=\mathcal{V}_{s} \mathcal{D}_{s} \mathcal{V}_{s}^{t}
$$

$\mathcal{V}_{s}$ is orthogonal, $\mathcal{D}_{s}$ is diagonal, and a solution reads

$$
\mathcal{P}=\mathcal{V}_{s}^{t}
$$

provided that

$$
\mathcal{V}_{s}^{t} \mathcal{E} \mathcal{V}_{s}=\mathcal{D}_{s}^{-1}
$$

Focusing on cantilever beams under flexure loading, $\mathcal{V}_{s}$ can be computed using a single test displacement field $\mathbf{U}_{m}^{\star}$. Defining

$$
\begin{aligned}
\mathbf{Z}_{1} & =\frac{\mathcal{E} \mathcal{V}^{t} \mathbf{U}_{m}^{\star}}{\left\|\mathcal{E} \mathcal{V}^{t} \mathbf{U}_{m}^{\star}\right\|} \\
\mathbf{Z}_{2}\left(\mathbf{r}_{0}\right) & =\frac{\mathcal{V}^{t}\left(\mathbf{U}_{m}^{\star}+\mathcal{R} \mathbf{r}_{0}\right)-\mathbf{Z}_{1}^{t} \mathcal{V}^{t}\left(\mathbf{U}_{m}^{\star}+\mathcal{R} \mathbf{r}_{0}\right) \mathbf{Z}_{1}}{\left\|\mathcal{V}^{t}\left(\mathbf{U}_{m}^{\star}+\mathcal{R} \mathbf{r}_{0}\right)-\mathbf{Z}_{1}^{t} \mathcal{V}^{t}\left(\mathbf{U}_{m}^{\star}+\mathcal{R} \mathbf{r}_{0}\right) \mathbf{Z}_{1}\right\|}
\end{aligned}
$$

the problem turns to find $\gamma$ and $\mathbf{r}_{0}$ so that

$$
\mathcal{V}_{s}^{t}=\left[\begin{array}{c}
\cos (\gamma) \sin (\gamma) \\
-\sin (\gamma) \cos (\gamma)
\end{array}\right]\left[\begin{array}{c}
\mathbf{Z}_{1}^{t} \\
\mathbf{Z}_{2}^{t}\left(\mathbf{r}_{0}\right)
\end{array}\right]
$$


satisfies Eq.(22) under the constrain defined by Eq.(26). It can be proved that the set

$$
\begin{aligned}
& \mathbf{r}_{0}=\mathbf{0} \\
& \gamma=\frac{1}{2} \tan ^{-1}\left(\frac{2 \mathbf{Z}_{2}(\mathbf{0})^{t} \mathcal{E} \mathbf{Z}_{1}}{\mathbf{Z}_{1}^{t} \mathcal{E} \mathbf{Z}_{1}-\mathbf{Z}_{2}(\mathbf{0})^{t} \mathcal{E} \mathbf{Z}_{2}(\mathbf{0})}\right)
\end{aligned}
$$

satisfies Eq.(22) and Eq.(26). The above described procedure can be repeated to allow Eq.(23) to be satisfied by $\frac{P}{2}$ distinct $\mathbf{U}_{m}^{\star}$ test displacement fields, considering orthogonal subspaces. It's worth noting the strain energy computed from the micro and macro displacement fields are then equal for the chosen $\mathbf{U}_{m}^{\star}$.

\subsection{Example}

The above described procedure is illustrated using a cantilever beam meshed by 8 elements on which the displacement is projected onto cubic Hermite functions (four degrees of freedom per element). The cantilever is clamped at one end and is free at the other. Numbering the elements from $n=1$ to $n=8$ when moving from the clamping to the free end, the stiffness is assumed to be constant on each element and to linearly decrease when moving away of the clamping, so that the bending stiffness of the element $n$ reads

$$
(E I)_{n}=(E I)_{0}\left(0.9-\frac{n}{10}\right)
$$

The cantilever is assumed to be loaded by a single transverse force applied to the last node.
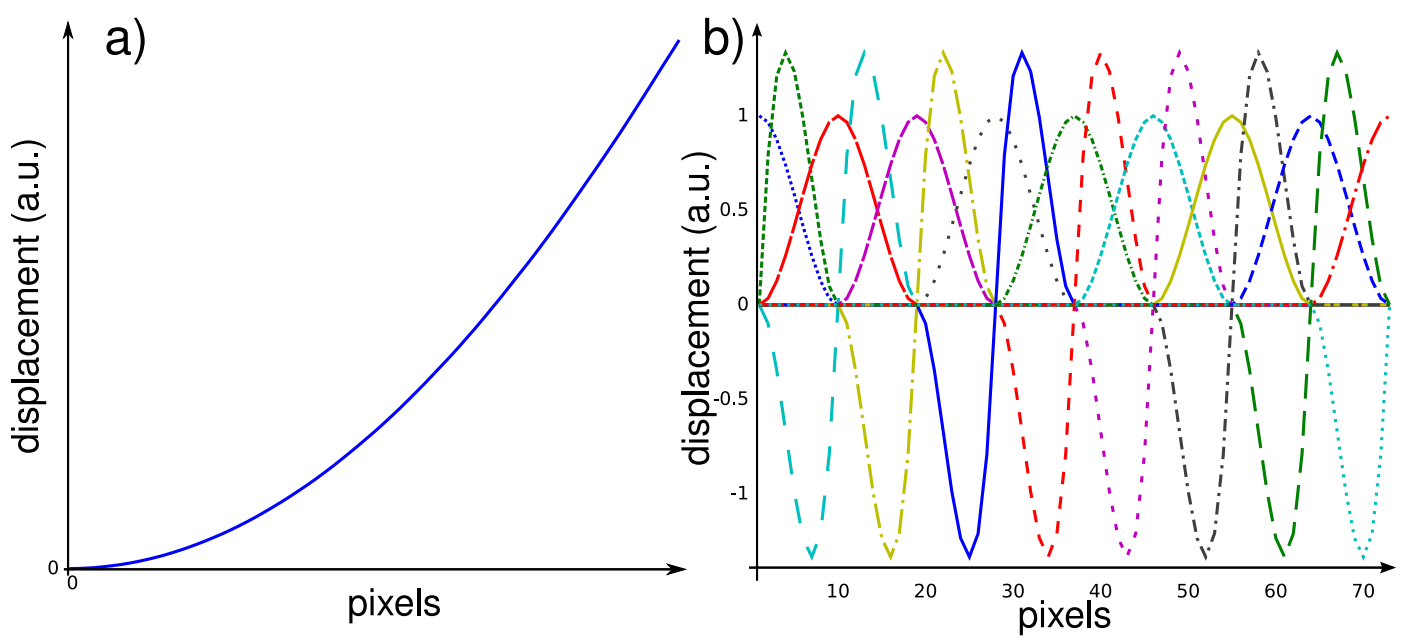

Fig. 1. a) Micro-scale displacement field used as an example b) Micro-displacement field basis.

The resulting displacement field (see Fig.1a) is described by 18 degrees of freedom. This displacement field basis is used as the micro-scale displacement fields basis (see Fig.1b) and is then used to examplify the above defined procedure. The first macro displacement fields basis is obtained using 4 dofs. Two test displacement fields are therefore used, namely $\mathbf{U}_{m}^{\star}=\widehat{\mathbf{U}_{m}}$ and $\mathbf{U}_{m}^{\star}=\mathbf{V}_{18}$ where $\mathbf{V}_{18}$ is the last column of $\mathcal{V}$, that is the dominant mode of the covariance matrix.

Fig. 2a shows the obtained displacement fields basis for 4 macro degrees of freedom. These macro degrees of freedom are those to be used when writing the equilibrium gap matrix at the macro scale, thus limitimg the effect of the measurement noise. Considering two additional macro dofs, the displacement fields basis shown on Fig. $2 \mathrm{~b}$ is obtained by adding $\mathbf{U}_{m}^{\star}=\mathbf{V}_{17}$. It can be shown this second, richer basis includes the basis obtained with 4 dofs, thus illustrating the construction of displacement fields basis families from the proposed method. 

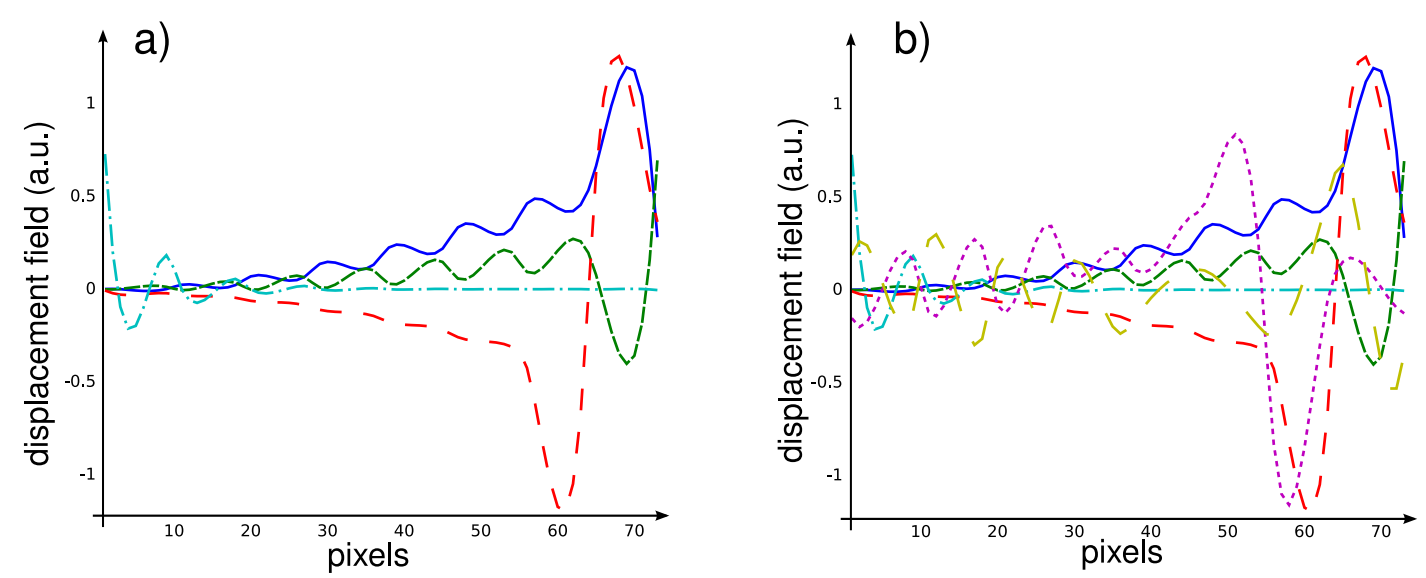

Fig. 2. a) Macro-scale displacement field basis obtained using a single macro element b) Macro-scale displacement field basis obtained using 2 macro elements.

\section{Conclusion}

A two-scales approach for the equilibrium gap method is presented. The projection conditions to be satisfied by the macro displacement field basis have been derived, and a procedure to build the projection operator is proposed. Various strategies can then be drawn in order to get the more robust identification procedure.

\section{References}

1. Bornert M., Doumalin P., Dupré J.-C., Fazzini M., Grédiac M., Hild F., Mistou S., Molimard J., Orteu J.-J., Robert L., Surrel Y., Vacher P. and Wattrisse B., Experimental Mechanics 49, (2009) 353-370.

2. Avril S., Bonnet M., Bretelle A.-S., Grédiac M., Hild F., Ienny P., Latourte F., Lemosse D., Pagano S., Pagnaco E., Pierron F., Experimental Mechanics 48, (2008) 381-402.

3. Amiot F., Hild F., Roger J.P., Int. J. SolidsStruct. 44, (2006) 2863-2887.

4. Grediac M., C. R. Acad Sci. Paris 309 [Serie II], (1989) 1-5.

5. Claire D., Hild F. and Roux S., C. R. Mecanique 330, (2002) 729-734. 